\title{
Levantamento das Estruturas Organizacionais em Organizações Autônomas Descentralizadas Baseadas em Blockchain
}

\author{
Alan Veloso ${ }^{1,2}$, Leonardo da Costa ${ }^{1}$, Billy Pinheiro ${ }^{1}$, \\ Antônio Abelém ${ }^{1}$, Filipe Saraiva ${ }^{2}$ \\ ${ }^{1}$ Grupo de Redes de Computadores e Comunicação Multimídia (GERCOM) \\ Universidade Federal do Pará (UFPA) - Belém - Pará - Brasil \\ ${ }^{2}$ Laboratório de Inteligência Artificial Aplicada (LAAI) \\ Universidade Federal do Pará (UFPA) - Belém - Pará - Brasil \\ alan.velosodicen.ufpa.br \\ $\{l b c, b i l l y$, abelem, saraiva\}@ufpa.br
}

\begin{abstract}
Blockchain-based Decentralized Autonomous Organizations (BC$D A O)$ are systems that perform security-based decision making. The organizational structure is an aspect that can contribute to its efficiency (e.g., by reducing redundant expenditure of resources, efforts and time). However, this aspect is not explicit in the development of BCDAO. This study presents a survey of organizational structures based on the literature on Multiagent System (MAS) and BCDAO, which include hierarchy, holarquia, society and markets. The study describes each of the organizational structures and how they are employed in $B C D A O$, in order to facilitate the comparative assessment of organizational styles. With this, the objective is to allow designers to know the current spectrum of possibilities and, then, guide the selection of an appropriate organizational structure for a particular application domain.
\end{abstract}

Resumo. As Organizações Autônomas Descentralizadas Baseadas em Blockchain (Blockchain-Based Decentralized Autonomous Organizations - BCDAO) são sistemas que realizam tomadas de decisões visando a segurança. A estrutura organizacional é um aspecto que pode contribuir para a eficiência (e.g., reduzindo gastos redundantes de recursos, esforços e tempo), no entanto, este aspecto não é explicito no desenvolvimento de BCDAO. Neste estudo é apresentado um levantamento das estruturas organizacionais baseadas na literatura de Sistema Multiagente (Multiagent System - MAS) BCDAO, que incluem hierarquia, holarquia, sociedade e mercados. O estudo descreve cada uma das estruturas organizacionais e como elas são empregadas em BCDAO, com o intuito de facilitar a avaliação comparativa de estilos organizacionais. Com isso, objetiva-se permitir a projetistas conhecer o espectro de possibilidades atual $e$, então, guiar a seleção de uma estrutura organizacional apropriada para um domínio de aplicação particular.

\section{Introdução}

A tecnologia blockchain, que é a base da moeda digital Bitcoin [Nakamoto 2008], fornece os meios para armazenar transações de forma distribuída e transparente, através de 
um conjunto de nós em rede [Swan 2015]. A blockchain Ethereum [Wood 2014] inovou ao possibilitar o envio e execução de contratos inteligentes nesta rede. Esses contratos surgiram com o objetivo de trazer versatilidade para as transações na blockchain, além de simples transferências de valores. Inicialmente, a intenção dos contratos inteligentes era de automatizar os termos de um contrato tradicional [Szabo 1997]. Contudo, esses contratos passaram a ser utilizados como códigos persistidos e distribuídos pela blockchain. Um dos seus empregos de maior destaque são as Organizações Autônomas Descentralizadas (Decentralized Autonomous Organization - DAO) [Dilger 1997].

A literatura fornece duas definições de DAO: A primeira, proposta por Jentzsch [Jentzsch 2016], define essas organizações como sendo constituídas por programas de computadores (contratos inteligentes no contexto de blockchain) utilizados para automatizar as regras de governança e a tomada de decisão da organização. A segunda definição, fornecida por Dilger [Dilger 1997], é originária de sistemas multiagentes (SMA) estabelece que essas organizações são agentes artificiais que interagem entre si, ou seja, um sistema multiagente, que detém parte ou toda a autoridade de tomada de decisão para alcançar o objetivo organizacional. Nesta última, os contratos inteligentes representam os agentes.

No presente estudo, DAO são compreendidas como sistemas multiagentes, pois é um campo de estudo consolidado e com estudos que podem ser utilizados como base. Essa abordagem, diferente da primeira, não considera um único contrato como uma organização, pois, como Puranam et al. [Puranam et al. 2014] definem, é necessário mais de um membro para haver uma organização. Neste estudo, as DAO no contexto de blockchain são nominadas como Organizações Autônomas Descentralizadas Baseadas em Blockchain (Blockchain-Based Decentralized Autonomous Organization - BCDAO).

Atualmente, os trabalhos relacionados a contratos inteligentes estão direcionados a aspectos individuais, como o de Worley e Skjellum [Worley and Skjellum 2019], não focando em macro-aspectos, como a estrutura organizacional que os contratos estão configurados. Entretanto, a estrutura organizacional também é importante para eficiência da organização, pois pode ser utilizada para limitar o escopo das interações, reduzir ou aumentar explicitamente a redundância do sistema, formalizar objetivos de alto nível ou impor certos mecanismos de coordenação para execução eficiente de uma tarefa [Horling and Lesser 2004].

Neste contexto, este trabalho pretende responder a seguinte questão de pesquisa: baseado na literatura de SMA, quais são as estruturas organizacionais presentes no campo de BCDAO atualmente? A resposta dessa pergunta visa contribuir com um conjunto de estruturas organizacionais que pode auxiliar o estudo e desenvolvimento de BCDAO, possibilitando orientar os interessados a selecionar uma estrutura organizacional mais apropriada para um domínio particular, a partir do melhor conhecimento de possibilidades das estruturas organizacionais disponíveis. A fim de alcançar o objetivo deste trabalho, foi necessário uma pesquisa bibliográfica de estruturas organizacionais presentes na literatura de MAS, bem como estudos e documentos sobre BCDAO existentes atualmente.

Este artigo está estruturado da seguinte maneira: a Seção 2 apresenta uma visão geral dos trabalhos que se relacionam com esse estudo. A Seção 3 descreve a metodologia utilizada. Subsequentemente, a Seção 4 descreve cada um das estruturas organizaci- 
onais encontrados e como elas estão contidas nas BCDAO e para finalizar, a Seção 5 faz considerações sobre este trabalho e apresentam alguns possíveis trabalhos futuros.

\section{Trabalhos Relacionados}

Como mencionado, o conceito de DAO não é recente. Apesar disso, no melhor do nosso conhecimento, ainda há uma carência de estudos direcionados a esse campo como um todo. Neste contexto, essa seção apresenta uma visão geral dos trabalhos relacionados a este estudo.

Slockit [Slockit 2016] propõem um framework para o desenvolvimento de BCDAO. A proposta foi concebida a partir de uma BCDAO funcional, a The DAO [Jentzsch 2016], que foi desenvolvida em Solidity, uma das linguagens de programação para contratos inteligentes da blockchain Ethereum. Considera-se a implementação do framework um ponto de destaque desse trabalho. Além de que, por ser concebido a partir de uma BCDAO operacional, os aspectos individuais dos contratos inteligentes e estrutura organizacional estão determinados. Essa característica possibilita que um conjunto de BCDAO que apresentam propósitos similares faça proveito quase integral do que já foi desenvolvido. Entretanto, para BCDAO que diferem em termos de propósito, esse framework pode não ser flexível o suficiente, tendo-se, assim, a necessidade de desenvolver as BCDAO do início.

Worley e Skjellum [Worley and Skjellum 2019] apresentam um conjunto de padrões de projetos para contratos inteligentes. A proposta deles é mais flexível, pois não fornece somente uma alternativa para os desenvolvedores (como é o caso do framework de Slockit) mas sim um conjunto de padrões para diferentes propósitos. Entretanto, esses padrões são direcionados para contratos em blockchains de uma maneira geral, sem estarem direcionados para BCDAO. Consequentemente, não há preocupação com a estrutura que esses contratos assumem em organizações, além de não possuírem implementação em uma linguagem de programação para uma blockchain, ficando a cargo do desenvolvedor aplicá-los.

A contribuição do presente estudo é apresentar um levantamento das estruturas organizacionais direcionado à $\mathrm{BCDAO}$ de diferentes propósitos, tendo em vista as $\mathrm{BC}$ DAO desenvolvidas atualmente. Contudo, em contraste com a contribuição de Worley e Skjellum, este trabalho não trata de aspectos de contratos inteligentes individuais, além de não ter implementação, como o framework proposto por Slockit.

\section{Metodologia}

Na primeira parte do estudo, foi realizada uma análise sistemática das estruturas organizacionais, que consistiu das seguintes etapas: primeiramente, a análise de cada estrutura organizacional individualmente possibilitou gerar a coleta das características de cada uma delas; posteriormente, foi realizada uma análise para unir características que se correlacionam com o intuito de uniformizar as características. As Tabelas 1 e 2 sintetizam a relação entre as características e os paradigmas encontrados neste estudo após a união realizada. A primeira apresenta as características das unidades na organização, enquanto a segunda apresenta as características da organização como um todo.

A segunda parte do estudo consistiu do levantamento das BCDAO, que tratou primeiramente de uma pesquisa bibliográfica para encontrar estudos primários sobre $\mathrm{BC}$ - 


\begin{tabular}{lllllllll}
\hline $\begin{array}{l}\text { Paradigmas } \\
\text { Estruturais }\end{array}$ & Unidade & Objetivo & Benefício & Relação & $\begin{array}{l}\text { Coordenação das } \\
\text { Atividades }\end{array}$ & Autonomia & Fluxo de controle & Fluxo de dados \\
\hline Hierarquia & Agente & Comum & Organização & Cooperam & Especificado & Parcial & Unidirecional & Multidirecional \\
\hline Holarquia & Holón & Comum & Organização & Cooperam & Especificado & Parcial & Unidirecional & Multidirecional \\
\hline Mercado & Agente & Particular & Individual & Competem & Intermediário & Total & Ausente & Multidirecional \\
\hline Sociedade & - & Individual & $\begin{array}{l}\text { Cooperam ou } \\
\text { Competem }\end{array}$ & - & Total & Total & - & - \\
\hline
\end{tabular}

Tabela 1: Relação entre Paradigmas Estruturais e Características (Unidade)

\begin{tabular}{|c|c|c|c|}
\hline $\begin{array}{l}\text { Paradigmas } \\
\text { Estruturais }\end{array}$ & Objetivo de Formação & Duração & Tipo de Sistema \\
\hline Hierarquia & Único & - & Fechado \\
\hline Holarquia & Único & - & Fechado \\
\hline Mercado & - & - & Aberto ou Fechado \\
\hline Sociedade & Ambiente & Longa & Aberto \\
\hline
\end{tabular}

Tabela 2: Relação entre Paradigmas Estruturais e Características (Organização)

DAO nas bases de dados Google Acadêmico, IEEE Xplore, ACM Digital Library, Springer Link e ScienceDirect. Após isso, realizou-se a avaliação do título e resumo de cada estudo para selecionar os que abordavam BCDAO. A maioria dos estudos eram pequisas secundárias (mapeamento e revisões sistemáticas). Dessa forma, somente o estudo de Alimoglu e Ozturan [Alimoglu and Ozturan 2017] foi considerado um estudo primário sobre BCDAO. Portanto, para expandir o conjunto de BCDAO, uma pesquisa documental foi realizada, buscando principalmente por white papers. Com isso, realizou-se uma segunda análise das BCDAO para remover BCDAO que não se enquadravam na definição proposta por Digler [Dilger 1997], onde ele determina que para haver uma organização é necessário mais de um agente.

Na terceira parte, a análise sistemática dos estudos retornados na primeira parte (levantamento dos paradigmas em SMA) possibilitou definir as características a serem identificadas nas BCDAO (levantadas na segunda parte). O trabalho de Horling e Lesser [Horling and Lesser 2004] foi a principal referência utilizada para a identificação das características, pois ele sintetiza os estruturas organizacionais presentes em SMA.

As características que possibilitaram determinar a estrutura pertencem aos membros individualmente e à organização como um todo. As características individuais de cada membro são as seguintes: o tipo de relação que membros da organização mantém entre si (eles podem cooperar ou competir); a autonomia que os membros podem apresentar no desempenho das suas funções, que pode ser total (quando o membro pode agir de forma independente de outros membros) ou parcial (quando há a necessidade da comunicação entre eles); a coordenação das atividades do membros, que pode ser realizada por um intermediário ou as funções podem ser predefinidas e cada membro tem as suas especificadas; e a disposição dos membros, ou seja, a topologia, que é identificada através da verificação dos fluxos de controle e fluxos de dados entre os membros.

As características das organizações como um todo são: o objetivo de formação, que pode ser apenas um e, no caso especifico de sociedades, funciona como ambiente; a duração, que é uma característica específica de sociedades, as quais devem ter longa duração; e o tipo de sistema, que pode ser aberto, se houver autonomia para os membros entrarem e saírem, ou fechado, caso contrário. A seguir, aborda-se como cada uma das características é encontrada nos estruturas organizacionais de BCDAO. 


\section{Resultados}

O estudo de Horling e Lesser [Horling and Lesser 2004] é a principal referência no tema de estruturas organizacionais e é base das subseções a seguir. Também é apresentado uma análise de cada estrutura organizacional e como estão presentes nas BCDAO atualmente. Realiza-se a descrição de três BCDAO (The DAO, Bitnation e Ethereum) que abrangem todo o espectro de estruturas organizacionais identificadas nas BCDAO levantadas. A escolha delas foi feita considerando o grau de relevância e notoriedade no contexto de BCDAO.

\subsection{Estruturas Organizacionais}

A estrutura organizacional é a coleção de comportamentos, relacionamentos e estrutura de autoridade que governam a organização, norteando os membros sobre como eles devem interagir entre si. Essas orientações podem influenciar na autoridade, fluxo de informações, alocação de recursos, padrões de coordenação e outras características do sistema [Hayden et al. 1999]. Os aspectos estruturais têm influência no desempenho de curto e longo prazo em organizações [Rodriguez et al. 2011]. A seguir, apresenta-se brevemente cada um das estruturas organizacionais encontrados nas BCDAO analisadas nesse estudo.

\subsubsection{Hierarquia}

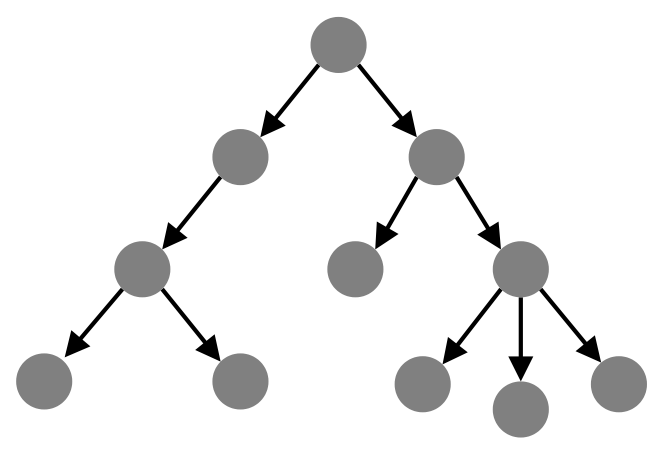

Figura 1. Hierarquia (adaptado de [Horling and Lesser 2004])

Na hierarquia, os membros são dispostos na estrutura de árvore, onde os de níveis mais altos têm uma visão geral dos processos abaixo deles (Figura 1). Na interpretação clássica da hierarquia, as interações são estabelecidas apenas entre entidades diretamente conectadas, o que significa que elas não cruzam a estrutura da árvore, ou seja, para comandos ou informações chegarem a um determinado membro será necessário passar por membros intermediários no caminho. Para fornecer a visão geral para os membros de níveis mais altos, os dados de agentes de níveis inferiores percorrem cada nível de baixo para cima, enquanto os fluxos de controle percorrem o sentido inverso, onde níveis a cima enviam comandos aos membros em níveis inferiores [Bond 1988].

Nesse modelo clássico, Fox [Fox 1979] ainda aponta 3 tipos de hierarquias, diferenciadas pela localização da autoridade de decisão e utilização de linhas de produto! Um dos tipos é a hierarquia simples, onde existe apenas um membro no topo da árvore com autoridade para tomar decisões. Outro é a hierarquia uniforme, onde tenta-se obter

\footnotetext{
${ }^{1}$ Produtos com funcionalidades similares
} 
ganhos de eficiência distribuindo a autoridade de tomada de decisão para membros dos demais níveis da árvore. Na hierarquia multidivisional, a hierarquia é dividida em linhas de produtos, onde os produtos podem representar artefatos físicos, serviços ou metas de alto nível. Cada divisão tem completo controle sobre o seu produto, o que facilita os processos de alocação de recursos e tomadas de decisão, visto que limita-se as influências externas.

\subsubsection{Características}

Em hierarquias, as unidades que as constituem são indivíduos e, no caso de BCDAO, estes são contratos inteligentes. A relação que os indivíduos mantém é de cooperação, buscando benefícios para a organização como um todo. Os contratos inteligentes seguem diferentes tipo de autonomia de acordo com a categoria de hierarquia: na simples, como a autoridade de tomada de decisão está concentrada no indivíduo que está no topo, a autonomia não existe para os outros membros; na uniforme, a autonomia é parcial, pois, apesar da tomada de decisão ser distribuída, ainda é necessário que indivíduos subordinados se reportem aos seus superiores; na multidivisional, a autonomia também é parcial, visto que os membros das linhas de produtos precisam se reportar aos seus superiores, apesar de as linhas de produção possuírem autonomia. As funções dos indivíduos são definidas previamente, visando evitar a redundância de funções. O fluxo de controle para um membro da organização vem de um único superior, enquanto esse mesmo superior pode ter mais de um subordinado, tendo fluxo de dados vindo de várias direções.

Hierarquias são constituídas com um único objetivo definido, portanto seus indivíduos irão desempenhar funções para alcança-lo. O tipo de sistema é fechado e o tempo de duração não é definido.

\subsubsection{Exemplo}

The DAO [Jentzsch 2016] é a BCDAO mais popular na literatura. Ela foi um marco que impulsionou pesquisas e desenvolvimentos relacionados a BCDAO. O principal objetivo era ser uma organização para intermediar financiamentos coletivos, similar ao Kickstartert

The DAO também funcionava como uma hierarquia (mais precisamente, uma hierarquia uniforme) após uma proposta ser aprovada. A proposta aprovada podia ser desenvolvida através de outros contratos inteligentes, o que possibilitava a eles serem parametrizados para receber fluxo de controle do contrato da The DAO. Contudo, a proposta ainda teria autonomia de funcionamento, ou seja, uma autonomia parcial. Como mais de uma proposta poderia ser aprovada, The DAO teria mais de um contrato para gerenciar e receberia diferentes fluxos de dados desses diferentes contratos.

\subsubsection{Holarquia}

As holarquias são estruturas que exploram as relações entre hólons [Koestler 1968], que são unidades completas, mas ao mesmo tempo parte de um todo maior (Figura 2). Em holarquias, cada unidade tem um caráter derivado, mas é distinto de outras. Cada hólon contribui para as propriedades de um ou mais hólons acima dele. Assim, cada hólon existe simultaneamente como uma entidade distinta, construída para englobar partes, e como parte de uma entidade maior.

\footnotetext{
2“Kickstarter." https: / / www. kickstarter.com/, Acessado em 21 ago. 2019.
} 


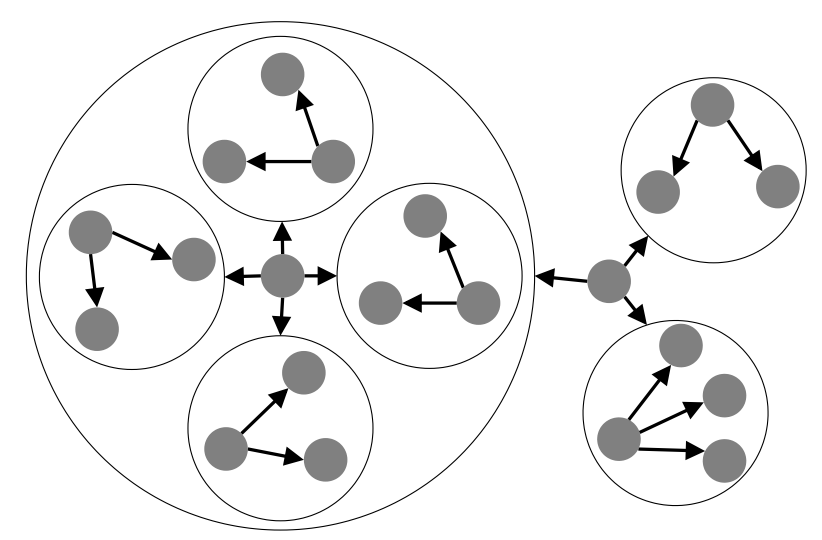

Figura 2. Holarquia (adaptado de [Horling and Lesser 2004])

A principal característica de uma holarquia é a parcialidade da autonomia dos hólons. Como cada uma dessas unidades é composta de um ou mais hólons subordinados e podem ser membro de um ou mais superordenados, o grau de autonomia atribuída a um hólon pode diferir entre os níveis ou mesmo entre similares no mesmo nível. Assim, a autonomia local de cada hólon não é completa, mas também não é totalmente ausente.

As holarquias ainda podem ter os membros estruturados pela divisão explícita ou implícita do trabalho [Ulieru et al. 2001]. Sendo assim, é necessário criar abstrações dessas divisões através da atribuição de recursos a hólons em vez de membros individuais. Essa camada de abstração permite que outras entidades na organização façam uso efetivo dos recursos, interagindo com uma única unidade funcional.

\subsubsection{Características}

As holarquias são similares às hierarquias. Entretanto, um dos diferenciais são as unidades que constituem esse tipo de estrutura, chamadas de holóns. Assim como nas hierarquias, as unidades colaboram para obter benefícios para a organização como um todo. A autonomia das unidades assemelha-se à autonomia da hierarquia multi-divisional, ou seja, os holóns são parcialmente autônomos. Nesse caso, há hólons que compõem outros hólons, estando subordinados a eles. A função de cada holón é definida previamente. Contudo, quando um holón recebe uma tarefa para ser realizada, ele tem autonomia de como proceder para executá-la. O fluxo de dados e de controle ocorre da mesma forma que em hierarquias, unidirecional de seu superior e multidirecional dos subordinados. A diferença é que, na hierarquia, o fluxo ocorre entre indivíduos e, na holarquia, ocorre entre holóns.

As características das organizações que utilizam a estrutura de holarquias são as mesmas que as de hierarquias: único objetivo, sistema fechado e tempo de duração não definido.

\subsubsection{Exemplo}

A Bitnation cria a estrutura de horlarquia dinamicamente. O hólon gênesis é criado e os membros - contratos inteligentes ou pessoas - desse hólon são responsáveis pelas decisões executivas sobre a direção estratégica da Bitnation. Os membros são selecionados com base no compromisso com a Bitnation, experiência em áreas designadas de trabalho e desempenho ao longo do tempo. Os outros hólons são formados para um fim específico, 


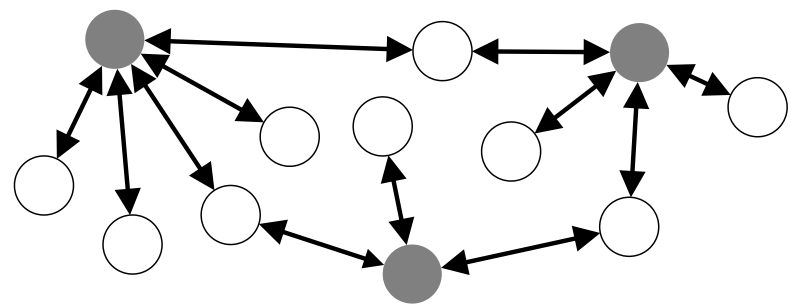

Figura 3. Mercado (adaptado de [Horling and Lesser 2004])

como o marketing da Pangea, ou completamente desconectado da operação da Bitnation, digamos um hólon trabalhando na organização de eventos sociais.

Todos os membros do Bitnation têm um campo de trabalho. Isso permite que os usuários tenham maior influência sobre as decisões se a proposta se referir ao seu campo de trabalho na organização. Existem vários signatários, cada um deles com uma participação específica em seu campo de trabalho (hólon), sendo eles responsáveis por sugerir orçamentos e propostas, além de revisar propostas. Assim, cada signatário opera como um centro de custo próprio, com maior autonomia.

\subsubsection{Mercado}

Em mercados há dois tipos de participantes, compradores e vendedores. Compradores podem solicitar ou dar lances para um conjunto comum de itens, como recursos compartilhados, tarefas, bens e serviços, enquanto vendedores oferecem itens para serem vendidos no mercado (Figura 3). Vendedores também podem delegar a terceiros a responsabilidade de processar lances e determinar o vendedor. Essa terceira parte recebe o nome de leiloeiro. Pela similaridade com sistemas de mercados do mundo real [Wellman 2004], resultados de pesquisas realizados nessa área podem ser aplicadas em mercados baseados em agentes [Corkill and Lander 1998].

Nessa estrutura, o vendedor é um membro distinto responsável por coordenar as atividades de um número de outros participantes compradores. Os compradores competem entre si para conseguir o item ofertado. Esses compradores confiam e cumprem as decisões do vendedor. Mercados podem operar tanto como um sistema fechado, onde a quantidade de vendedores e compradores é determinado, quanto como um sistema aberto, o que possibilita a qualquer agente participar, desde que ele respeite as regras especificadas pelo sistema.

\subsubsection{Características}

Indivíduos (i.e., contratos inteligentes) são as únicas unidades presentes nas organizações que implementam mercado. Os indivíduos, também chamados de compradores, intrinsecamente competem para obter um determinado item, ou seja, os objetivos das unidades são individuais, diferente das estruturas descritas anteriormente, onde as unidades buscam benefícios para a organização como um todo. A autonomia dessas unidades é total. Entretanto, existe um ente diferenciado, o vendedor, que coordena as atividades dos compradores. Apesar disso, o vendedor não impõe controle sobre os compradores, mas somente administra a venda do item e determina quem conseguiu obtê-lo. O vendedor deverá estar apto para receber múltiplos fluxos de dados dos diferentes compradores. 


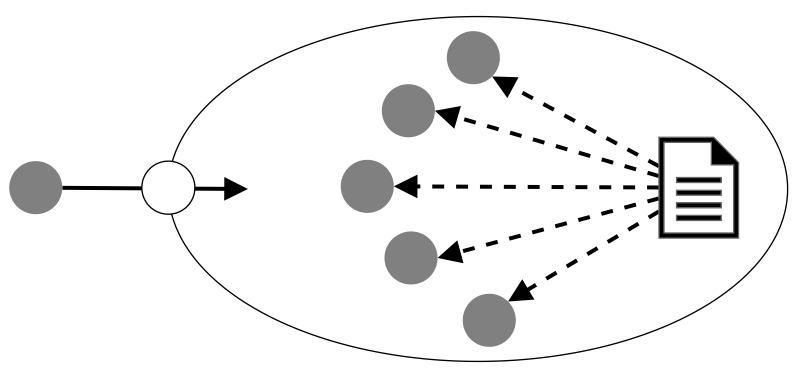

Figura 4. Sociedade (adaptado de [Horling and Lesser 2004])

Organizações que apresentam esse tipo de estrutura podem ser tanto em sistemas fechados quanto em sistemas abertos. Em sistemas abertos, onde compradores podem entrar e sair da disputa, é importante que o indivíduo responsável por gerenciar a venda dos itens desempenhe de forma eficiente essa habilidade para que não haja erros, como, por exemplo, determinar equivocadamente que um comprador que saiu da disputa obteve um item.

\subsubsection{Exemplo}

Pela necessidade de levantar capital, The DAO funciona como um mercado. Ela oferece tokens como produto para os compradores. Esses tokens garantem benefícios para seus detentores, como a possibilidade de votar nas propostas recebidas pela organização. Um limite pré-estabelecido de tokens é ofertado. Portanto, os compradores disputam por eles. A coordenação das compras dos tokens é feita pelo contrato da The DAO, internamente associando os tokens às contas dos compradores. Além disso, The DAO é um sistema aberto, pois não há restrição de quem pdoe comprar os tokens.

Para atingir os objetivos, The DAO delega a terceiros processos para os quais ela não despõem de aptidão para realizar. Para este fim, ela funciona como um mercado reverso. The DAO recebe propostas de projetos de qualquer interessado. Esses interessados podem ser até mesmo os próprios compradores de tokens. Novamente, há a competição dos compradores para terem suas propostas aprovadas, enquanto a avaliação das propostas é feita pelos detentores de tokens.

\subsubsection{Sociedade}

Diferente de outros estruturas organizacionais, sociedades são, inerentemente, sistemas abertos. Elas funcionam como um ambiente, fornecendo um domínio comum onde os participantes podem atuar e interagir. As sociedades estabelecem a estrutura e a ordem, mas as composições das interações podem ser bastante flexíveis (Figura 4). Nessa estrutura, os participantes podem compor diferentes organizações ou serem completamente independentes.

Uma das principais características que distinguem sociedades de outras estruturas organizacionais são as leis, normas ou convenções, que impõem restrições ao comportamento dos membros da mesma. Essas restrições tem por objetivo harmonizar o comportamento para facilitar a coexistência dos integrantes da sociedade. Por exemplo, elas podem ser o protocolo utilizado na comunicação, a moeda utilizada em negociações ou limitações no comportamento que o agente pode desempenhar no ambiente. As leis determinadas por uma sociedade devem ser justas, ou seja, não devem beneficiar os objetivos 
de uma classe de indivíduos em detrimento de outras.

\subsubsection{Características}

Sociedades funcionam como ambientes. Isto significa que suas unidades podem ser tanto indivíduos quanto organizações. Cada uma dessas unidades tem um objetivo particular. Elas podem cooperar ou competir, se os processos para alcançar seus objetivos por algum motivo se relacionarem. Apesar disso, cada uma dessas unidades tem autonomia total sobre si, sem a necessidade de prestar contas a outro ente pertencente a sociedade. Portanto, não há um membro que coordene as atividades dessas unidades. Entretanto, a sociedade pode impor indiretamente controle sobre as unidades através de leis, normas ou convenções sociais a respeito do modo com que cada indivíduo se relaciona com outro.

Sociedades apresentam longa duração para que suas unidades possam subsistir. Além disso, elas são inerentemente sistemas abertos, de onde qualquer unidade pode entrar e sair.

\subsubsection{Exemplo}

A blockchain do Bitcoin foi a primeira a ser desenvolvida. Entretanto, ela não apresenta a mesma importância que o Ethereum para o desenvolvimento de BCDAO, pois esta foi a primeira blockchain que possibilitou o envio de contratos inteligentes desenvolvidos por qualquer pessoa para a rede [Wood 2014]. Assim, por ser o precursor desse mecanismo, o Ethereum tornou-se popular para o desenvolvimento tanto de aplicações que utilizam blockchain (como DApps) quanto de BCDAO.

O Ethereum apresenta a estrutura de uma sociedade, pois possibilita a existência de contratos inteligentes individuais ou de outras BCDAO. Somente uma BCDAO apresentada no presente estudo, The DAO, não está mais executando sobre a blockchain Ethereum.

As unidades que subsistem na blockchain Ethereum podem ser contratos inteligentes individuais ou BCDAO. Essas unidades apresentam seus próprios objetivos individuais. No entanto, o processo para alcançar seus objetivos pode fazer com que se relacionem de modo competitivo ou cooperativo. Elas também possuem autonomia total para alcançarem seus objetivos. Contudo, o Ethereum ainda emprega controle indireto sobre as unidades (e.g., definindo a moeda utilizada).

Por se tratar de uma sociedade, o Ethereum funciona como um ambiente aberto e de longa duração para contratos inteligentes e BCDAO.

\subsection{Análise dos Resultados}

Anteriormente, foi descrito como as estruturas organizacionais estão presentes nas seguintes BCDAO: The DAO, Bitnation e Ethereum. Uma breve descrição sobre como as estruturas organizacionais são encontradas nessas organizações é sintetizada na Tabela 3 . Nessa tabela, pode-se observar que mais de uma estrutura organizacional é adotado para diferentes situações, pois algumas abordagens são mais adequadas que outras para determinadas situações [Horling and Lesser 2004]. Logo, a escolha da(s) estrutura(s) adotada(s) por um projetista deve ser direcionada pelos requisitos especificados para o sistema em questão.

Estruturas organizacionais são fundamentais para o desenvolvimento de BCDAO, 


\begin{tabular}{lll}
\hline $\begin{array}{l}\text { Organização Autônoma } \\
\text { Baseada em Blockchain }\end{array}$ & Paradigma Estrutural & \multicolumn{1}{c}{ Descrição } \\
\hline The DAO & Mercado & $\begin{array}{l}\text { Na fase inicial, para obter capital para financiar propostas, ela oferece tokens, o } \\
\text { que garantem proporcionalmente, o direito de voto aos contribuintes na seleção das } \\
\text { propostas a serem financiadas. Posteriormente, ao receber as propostas, atua como } \\
\text { mercado reverso. }\end{array}$ \\
\cline { 2 - 3 } & Hierarquia & $\begin{array}{l}\text { Após a aprovação de uma proposta, o contrato dela pode ser parametrizado } \\
\text { para receber influência da The DAO }\end{array}$ \\
\hline Ethereum & Sociedade & $\begin{array}{l}\text { Ambiente onde outros contratos inteligentes podem interagir, possibilita a criação } \\
\text { de contratos inteligentes, aplicações ou outras organizações. Determinar a moeda } \\
\text { utilizada e a forma de interação entre os contratos inteligentes. }\end{array}$ \\
\hline Bitnation & Holarquia & $\begin{array}{l}\text { Implementa uma holarquia líquida que possibilita aos seus usuários e contratos } \\
\text { inteligentes formarem hólons autônomos. }\end{array}$ \\
\hline
\end{tabular}

Tabela 3: Paradigmas Estruturais em Organizações Autônomas Baseadas em Blockchain

mesmo que de forma implícita. Corkill e Lander [Corkill and Lander 1998] enumeram vários outros fatores que motivam a necessidade de evidenciar a organização, incluindo recursos escassos, o potencial de colaboração e a quantidade de repetição de trabalho. Portanto, apresentar o espectro de possibilidades para projetistas de BCDAO é necessário para torná-las mais eficientes. Ao reconhecer e entender as estruturas organizacionais, como os deste trabalho, espera-se que o uso de projeto estrutural explícito seja incentivado e facilitado.

Horling e Lesser [Horling and Lesser 2004] também apresentam outras estruturas não verificadas nas BCDAO analisadas nesse artigo. Elas são: coalizão, time, congregação, federação e matriz. Apesar de não terem sido identificadas nas BCDAO, ainda há a possibilidade de serem utilizadas para desenvolver outras BCDAO. A coalizão é uma estrutura de curta duração que surge para alcançar um objetivo específico que está relacionado a um ou mais membros, onde esses membros precisam oferecer esforços para alcançar o objetivo. A coalizão se desfaz assim que o objetivo é alcançado. O time é similar à coalizão. A principal diferença entres eles é que, no primeiro, há a coordenação das tarefas de todos os membros para potencializar a finalidade do time e não objetivos individuais. A congregação assemelha-se à coalização, contudo, diferente desta onde os objetivos dos membros são semelhantes, os objetivos dos membros da congregação são geralmente diversificados. Além disso, uma congregação é uma formação de longa duração. Tipicamente, os indivíduos se juntam a uma congregação para obter benefícios, buscando encontrar membros que possuam características similares ou complementares.

Em organizações que apresentam a estrutura de federação, membros do grupo cedem parte da sua autonomia para um único membro que representa o grupo [Genesereth 1997]. Esse membro distinto geralmente recebe o nome de facilitador, mediador ou broker [Hayden et al. 1999]. Ele é responsável por intermediar a comunicação interna entre os membros e entre os membros e entes externos à organização. A matriz é similar à hierarquias, contudo, elas diminuem a restrição de um membro ter apenas um gerente, permitindo vários gerentes influenciando as atividades de um membro, isto é, os comandos podem vir de muitas direções, cada uma com seus próprios objetivos, importância e características [Wagner and Lesser 2000].

Outra estrutura também é apresentada por Horling e Lesser: a composta. Esta é uma estrutura híbrida, que compreende mais de uma estrutura. A maioria das organizações apresenta esse modelo. Entretanto, como um dos objetivos deste trabalho é 
distinguir os estruturas organizacionais, ela não foi considerada como uma estrutura isolada, mas sim como um combinação de outras estruturas. Ela é utilizada quando pretendese usufruir das diferentes características de diferentes estruturas organizacionais.

\section{Considerações Finais e Trabalhos Futuros}

Este estudo reportou o conjunto de estruturas organizacionais utilizadas atualmente em BCDAO e fundamentada na literatura de MAS, de modo a fornecer aos projetistas de BCDAO o espectro de possibilidades dessas estruturas (hierarquia, holarquia, mercado e sociedade). A análise das BCDAO também forneceu possíveis casos de uso de cada uma das estruturas encontradas, entretanto, o uso não é restrito ao propósito de cada uma.

Possíveis trabalhos futuros consistem em desenvolver padrões de projetos das estruturas organizacionais e desenvolver um framework em uma linguagem de programação de uma blockchain (e.g. Ethereum) considerando esses padrões. Também é possível desenvolver um estudo sobre os aspectos individuais de contratos inteligentes direcionados para BCDAO. Além disso, é possível expandir o padrões de projetos considerando as estruturas apresentadas na Seção 4.2 que não estão presentes atualmente nas BCDAO.

\section{Agradecimentos}

O presente trabalho foi realizado com apoio da Coordenação de Aperfeiçoamento de Pessoal de Nível Superior - Brasil (CAPES) - Código de Financiamento 001. Os autores agradecem o apoio da Universidade Federal do Pará (UFPA).

\section{Referências}

[Alimoglu and Ozturan 2017] Alimoglu, A. and Ozturan, C. (2017). Design of a smart contract based autonomous organization for sustainable software. Proceedings - 13th IEEE International Conference on eScience, eScience 2017, pages 471-476.

[Bond 1988] Bond, A. H. (1988). An analysis of problems and research in dai. Readings in Distributed Artificial Intelligence, pages 3-35.

[Corkill and Lander 1998] Corkill, D. D. and Lander, S. E. (1998). Diversity in agent organizations. Object Magazine, 8(4):41-47.

[Dilger 1997] Dilger, W. (1997). Decentralized autonomous organization of the intelligent home according to the principle of the immune system. In 1997 IEEE International Conference on Systems, Man, and Cybernetics. Computational Cybernetics and Simulation, volume 1, pages 351-356. IEEE.

[Fox 1979] Fox, M. S. (1979). Organization structuring: designing large complex software. Technical Report CMU-CS-79-155, Carnegie-Mellon Univ. Dept. Comput. Sci., Pittsburgh, PA.

[Genesereth 1997] Genesereth, M. R. (1997). An agent-based framework for interoperability. Software agents, 37(7):317-345.

[Hayden et al. 1999] Hayden, S. C., Carrick, C., and Yang, Q. (1999). Catalog of agent coordination patterns. Proceedings of the International Conference on Autonomous Agents, pages 412-413.

[Horling and Lesser 2004] Horling, B. and Lesser, V. (2004). A survey of multi-agent organizational paradigms. Knowledge Engineering Review, 19(4):281-316. 
[Jentzsch 2016] Jentzsch, C. (2016). Decentralized autonomous organization to automate governance. Technical report.

[Koestler 1968] Koestler, A. (1968). The ghost in the machine. Macmillan, Oxford, England.

[Nakamoto 2008] Nakamoto, S. (2008). Bitcoin: A Peer-to-Peer Electronic Cash System. Www.Bitcoin.Org, page 9.

[Puranam et al. 2014] Puranam, P., Alexy, O., and Reitzig, M. (2014). What's "New" About New Forms of Organizing? Academy of Management Review, 39(2):162-180.

[Rodriguez et al. 2011] Rodriguez, S., Julián, V., Bajo, J., Carrascosa, C., Botti, V., and Corchado, J. M. (2011). Agent-based virtual organization architecture. Engineering Applications of Artificial Intelligence, 24(5):895-910.

[Slockit 2016] Slockit (2016). Decentralized Autonomous Organization (DAO) Framework.

[Swan 2015] Swan, M. (2015). Blockchain: Blueprint for a new economy.

[Szabo 1997] Szabo, N. (1997). Formalizing and Securing Relationships on Public Networks. First Monday, 2(9).

[Ulieru et al. 2001] Ulieru, M., Walker, S. S., and Brennan, R. W. (2001). The Holonic Enterprise as a Collaborative Information Ecosystem. In Workshop on "Holons: Autonomous and cooperative Agents for the Industry."Autonomous Agents.

[Wagner and Lesser 2000] Wagner, T. and Lesser, V. (2000). Relating Quantified Motivations for Organizationally Situated Agents. In Jennings, N. R. and Lespérance, Y., editors, Intelligent Agents VI. Agent Theories, Architectures, and Languages, pages 334-348, Berlin, Heidelberg. Springer Berlin Heidelberg.

[Wellman 2004] Wellman, M. P. (2004). Online Marketplaces.

[Wood 2014] Wood, G. (2014). Ethereum: a secure decentralised generalised transaction ledger. Ethereum Project Yellow Paper, pages 1-32.

[Worley and Skjellum 2019] Worley, C. R. and Skjellum, A. (2019). Opportunities, Challenges, and Future Extensions for Smart-Contract Design Patterns. In Lecture Notes in Business Information Processing, volume 57, pages 264-276. Springer International Publishing. 